This item was submitted to Loughborough's Research Repository by the author.

Items in Figshare are protected by copyright, with all rights reserved, unless otherwise indicated.

\title{
Interaction of two lump solitons described by the Kadomtsev-Petviashvili I
} equation

PLEASE CITE THE PUBLISHED VERSION

LICENCE

CC BY-NC-ND 4.0

\section{REPOSITORY RECORD}

Lu, Zhiming, Emily M. Tian, and Roger H.J. Grimshaw. 2019. "Interaction of Two Lump Solitons Described by the Kadomtsev-petviashvili I Equation”. figshare. https://hdl.handle.net/2134/308. 


\title{
Loughborough University
}

This article has been submitted to Loughborough University's Institutional Repository by the author.

\section{Interaction of two lump solitons described by the Kadomtsev-Petviashvili I equation}

\author{
Zhiming $\mathrm{Lu}^{1}$, Emily M. Tian ${ }^{2}$ and Roger Grimshaw ${ }^{1}$ \\ ${ }^{1}$ Department of Mathematical Sciences, Loughborough University, \\ Loughborough, Leics, LE11 3TU, UK \\ ${ }^{2}$ Department of Mathematics and Statistics, Wright State University, \\ 3640 Colonel Glenn Hwy, Dayton, Ohio 45435, USA
}

June 4, 2003

\begin{abstract}
The interaction of two lump solitons described by the Kadomtsev-Petviashvili I(KPI) equation is analyzed using both exact and numerical methods. The numerical method is based on a third order Runge-Kutta method, and a Crank- Nicholson scheme. The main characteristic of a direct interaction when the two lumps are initially aligned along the $x$-axis, is that they may separate in the $y$-direction, but then come back to the $x$ axis after collision; the dependence of the maximum separation in the $y$-direction on the relative velocity difference is described. Two lumps may also experience an abrupt phase change in the case of an oblique interaction.
\end{abstract}

\section{Introduction}

The Kadomtsev-Petviashvili (KP) equation

$$
\frac{\partial}{\partial x}\left(\frac{\partial u}{\partial t}+6 u \cdot \frac{\partial u}{\partial x}+\frac{\partial^{3} u}{\partial x^{3}}\right)+3 \sigma^{2} \frac{\partial^{2} u}{\partial y^{2}}=0,
$$

is one of the prototype equations with wide applications in the modern physics of nonlinear waves[1, 3, 12]. In the case $\sigma^{2}=-1$, Eq. (1) is usually called the KPI equation, whereas in the case $\sigma^{2}=1$, the KPII equation. Both KPI and KPII are exactly integrable via the Inverse Scattering Transformation (IST). The characteristic features of solutions of the KP equation are essentially different for the choice of the sign of $\sigma^{2}$. Plane solitons (of the KdV equation) are stable with respect to a transverse disturbance for the KPII equation, but, they are unstable to a transverse disturbance for the KPI equation. At the same time, the KPI equation admits lump-type solitons, which are localized in all directions, and decay algebraically. Lump solitons have been widely investigated since they were first discovered[10, 14]. A striking property of the interaction of two lump solitons is that not only does each soliton retain its shape and initial parameters (amplitude, velocity, size) after collision, but its final phase shift also turns out to be zero (which is in marked contrast to the collision of two solitons of the KdV equation). But this does not mean 
that the interaction of two (or more) such solitons is as trivial as the superposition of their individual fields. Pelinovsky \& Stepanyants [16], Gorshkov et al. [10] considered multi-solitons and used asymptotic and exact methods to describe lump soliton dynamics. For the case of a direct interaction, when the solitons are initially aligned along the same $x$-direction, they may interact through a process of partial separation in the transverse $y$ direction, before returning to the $x$-axis; in particular, they found that when the asymptotic velocity difference (of two the solitons) vanishes, the nonlinear interaction leads to an infinite phase shift of their trajectories; further, for some multi-solitons there may exist equilibrium states corresponding to bound states of individual solitons. Ablowitz and Villarroel [2] also constructed this class of lump solitons using IST in combination with perturbation methods, and more properties of multi-solitons were found.

More recently, lump solitons have been found to exist in some other nonlinear equations $[3,7,9,17]$. However the dynamic behaviour of the interaction of multiple lump solitons so far has not been fully understood; moreover, there are examples of inexact, or quasi-soliton behaviour where no analytical results are available and thus numerical studies are essential to develop the understanding of the phenomena. In fact, the lump soliton and bound-state multi-lump-solitons were first observed numerically and then found theoretically [10].

The numerical analysis literature for the $\mathrm{KP}$ equation is relatively rare compared to the $\mathrm{KdV}$ equation. Among these, some schemes are based on the finite difference method $[4,5,6,13,18]$, while others $[3,11,15]$ are based on the pseudo-spectral method first developed by Fornberg and Whitham [8]. In [5] and [6], the authors found that two lumps with different amplitudes will separate in the $y$-direction with equal amplitudes in a direct interaction (but note that the theory tells us they will eventually return to the $x$-axis, see below). More recently, the oblique collision of two lumps of equal amplitude travelling from the opposite $y$-direction to the horizontal (which corresponds to oblique collision 1 in this study) and the generation of lump solitons by the bottom topography have been studied in terms of a generalised Benney-Luke equation taking into account the effects of surface tension and topographic forcing [3]. Nevertheless, the nonlinear interaction process of two lump solitons is far from clear and understood.

In this paper, we study the interaction of lump solitons using primarily numerical meth-

ods, supported by some theoretical results. The remainder of the paper is organised as follows: exact solutions for the interaction of multiple lump solitons are given in the next section, and the numerical methods adopted here are described briefly in section 3; the numerical experiments and comparison with theoretical results are in section 4; and finally the conclusion is in section 5 .

\section{Exact solutions of multiple lump solitons}

The exact multisoliton solutions of the KPI equation (Eq. 1) can be obtained in several ways. Here we write them in the Hirota form:

$$
u(x, y, t)=2 \frac{\partial^{2} \ln \phi}{\partial x^{2}}
$$

where $\phi$ is defined as

$$
\phi=\operatorname{det}\left[\left(x-2 k_{i \pm} y+12 k_{i \pm}^{2} t+\gamma_{i \pm}\right) \cdot \delta_{i, l}-j \cdot\left(1-\delta_{i, l}\right) /\left(k_{i \pm}-k_{l \pm}\right)\right],
$$


and $\delta_{i, l}$ is the Kronecker symbol. Here $i, l=1,2, \ldots, N \equiv 2 M$, and $M$ specifies the number of solitons. The parameters $k_{i \pm}=k_{i R} \pm j k_{i I}$ and $\gamma_{i \pm}=\gamma_{i R} \pm j \gamma_{i I}$ determine the amplitude (velocity) and the phase of each soliton. In particular, if $M=1$, then

$$
\phi=\left(\xi-2 k_{1 R} \eta\right)^{2}+4 k_{1 I}^{2} \eta^{2}+\frac{1}{4 k_{1 I}^{2}},
$$

and

$$
u(x, y, t)=16 \frac{-4\left(\xi-2 k_{1 R} \eta\right)^{2}+16 k_{1 I}^{2} \eta^{2}+\frac{1}{k_{1 I}^{2}}}{\left[4\left(\xi-2 k_{1 R} \eta\right)^{2}+16 k_{1 I}^{2} \eta^{2}+\frac{1}{k_{1 I}^{2}}\right]^{2}},
$$

where

$$
\xi=x-12\left(k_{1 R}^{2}+k_{1 I}^{2}\right) t+\gamma_{1 R}+\frac{k_{1 R} \gamma_{1 I}}{k_{1 I}}, \quad \eta=y-12 k_{1 R} t+\frac{\gamma_{1 I}}{2 k_{1 I}} .
$$

This is the lump soliton.

In this paper we are mainly concerned with the interaction of two lump solitons with different velocities and also different phases. Let $M=2$ we get

$$
\phi=\operatorname{det}\left(\begin{array}{cccc}
X_{1}-j Y_{1} & -\frac{1}{2 k_{1 I}} & -\frac{j}{k_{R}+j k_{I 1}} & -\frac{j}{k_{R}+j k_{I 2}} \\
\frac{1}{2 k_{1 I}} & X_{1}+j Y_{1} & -\frac{j}{k_{R}-j k_{I 2}} & -\frac{j}{k_{R}-j k_{I 1}} \\
\frac{j}{k_{R}+j k_{I 1}} & \frac{j}{k_{R}-j k_{I 2}} & X_{2}-j Y_{2} & -\frac{1}{2 k_{2 I}} \\
\frac{j}{k_{R}+j k_{I 2}} & \frac{j}{k_{R}-j k_{I 1}} & \frac{1}{2 k_{2 I}} & X_{2}+j Y_{2}
\end{array}\right)
$$

where

$$
\begin{array}{cc}
X_{1}=x-2 k_{1 R} y+12\left(k_{1 R}^{2}-k_{1 I}^{2}\right) t+\gamma_{1 R}, & Y_{1}=2 k_{1 I}\left(y-12 k_{1 R} t-\frac{\gamma_{1 I}}{2 k_{1 I}}\right), \\
X_{2}=x-2 k_{2 R} y+12\left(k_{2 R}^{2}-k_{2 I}^{2}\right) t+\gamma_{2 R}, & Y_{2}=2 k_{2 I}\left(y-12 k_{2 R} t-\frac{\gamma_{2 I}}{2 k_{2 I}}\right), \\
k_{R}=k_{1 R}-k_{2 R}, \quad k_{I 1}=k_{1 I}-k_{2 I}, & k_{I 2}=k_{1 I}+k_{2 I},
\end{array}
$$

It can be shown that the leading term of the polynomials $\phi$ is a product of the polynomials $\phi_{i}, i=1,2$ corresponding to individual solitons. In fact the solution (6) describes the interaction of two lump solitons of different velocities $V_{1}, V_{2}$, which are initially located at $\left(x_{01}, y_{01}\right)$ and $\left(x_{02}, y_{02}\right)$ respectively, where we have the components

$$
\begin{array}{llll}
V_{1 x}=12\left(k_{1 R}^{2}+k_{1 I}^{2}\right), & V_{1 y}=12 k_{1 R}, & x_{01}=\gamma_{1 R}+\frac{k_{1 R}}{k_{1 I}} \gamma_{1 I}, & y_{01}=\frac{\gamma_{1 I}}{2 k_{1 I}}, \\
V_{2 x}=12\left(k_{2 R}^{2}+k_{2 I}^{2}\right), & V_{2 y}=12 k_{2 R}, & x_{02}=\gamma_{2 R}+\frac{k_{2 R}}{k_{2 I}} \gamma_{2 I}, & y_{02}=\frac{\gamma_{2 I}}{2 k_{2 I}}
\end{array}
$$

From our numerical experiments and this exact solution, the interaction of two lump solitons can be classified into three types, i.e. the direct interaction in which the solitons are initially aligned along the $x$-axis (i.e. $V_{1 y}=V_{2 y}=0$ ), and two oblique interactions which are determined by the relative signs of $V_{1 y}$ and $V_{2 y}$. 


\section{Numerical methods of KPI equation}

Compared with the pseudo-spectral method, the finite difference method possesses some advantages such as simplicity, and being adjustable to non-regular computational domains. Many numerical experiments have shown that the finite difference method can often obtain the same accuracy as spectral methods. When we consider the numerical solution of KP equation, as pointed out by Katis and Akylas [13], it is convenient to integrate the equation once with respect to $\mathrm{x}$, then Eq. (1) reads:

$$
\frac{\partial u}{\partial t}+6 u \cdot \frac{\partial u}{\partial x}+\frac{\partial^{3} u}{\partial x^{3}}-3 \int_{-\infty}^{x} \frac{\partial^{2} u}{\partial y^{2}} \mathrm{~d} x=0,
$$

which can be written in the general form:

$$
\frac{\partial u}{\partial t}=N(u)+L(u)
$$

where

$$
N(u)=-6 u \cdot \frac{\partial u}{\partial x}+3 \int_{-\infty}^{x} \frac{\partial^{2} u}{\partial y^{2}} \mathrm{~d} x \quad L(u)=-\frac{\partial^{3} u}{\partial x^{3}}
$$

When choosing schemes to discretise a partial differential equation, one has to compromise between the time accuracy and the storage requirements. An efficient algorithm for the time integration for a partial differential equation is a low storage third-order Runge-Kutta explicit scheme. On the other hand, an implicit treatment for linear term $L(u)$ doesn't require any particular effort, and it has been widely acknowledged that the Crank-Nicolson implicit scheme is one of the most effective. By evaluating the linear term $L(u)$ at the time level $n+1 / 2$ it follows

$$
\frac{1}{2}\left(L^{\prime}\left(u^{n}\right)+L^{\prime}\left(u^{n+1}\right)\right)
$$

where the difference operator $L^{\prime}$ is defined as follows

$$
L^{\prime}=-\frac{u_{i+2}-2 u_{i+1}+2 u_{i-1}-u_{i-2}}{2 \triangle x^{3}},
$$

and $\triangle x$ denotes the spatial step in the $x$-direction and the subscript $j$ is omitted for briefness. Finally, we can get the scheme

$$
\begin{aligned}
u^{n+\frac{1}{3}} & =u^{n}+\gamma_{1} \triangle t N\left(u^{n}\right)+\alpha_{1} \triangle t \frac{L\left(u^{n+\frac{1}{3}}\right)+L\left(u^{n}\right)}{2} \\
u^{n+\frac{2}{3}} & =u^{n+\frac{1}{3}}+\gamma_{2} \triangle t N\left(u^{n+\frac{1}{3}}\right)+\rho_{1} \triangle t N\left(u^{n}\right)+\alpha_{2} \triangle t \frac{L\left(u^{n+\frac{2}{3}}\right)+L\left(u^{n+\frac{1}{3}}\right)}{2}, \\
u^{n+1} & =u^{n+\frac{2}{3}}+\gamma_{3} \triangle t N\left(u^{n+\frac{2}{3}}\right)+\rho_{2} \triangle t N\left(u^{n}\right)+\alpha_{3} \triangle t \frac{L\left(u^{n+1}\right)+L\left(u^{n+\frac{2}{3}}\right)}{2},
\end{aligned}
$$

where the coefficients are given by

$$
\begin{array}{rlrl}
\alpha_{1} & =\frac{8}{15}, & \alpha_{2}=\frac{2}{15}, & \alpha_{3}=\frac{1}{3}, \\
\gamma_{1}=\frac{8}{15}, & \gamma_{2}=\frac{5}{12}, & \gamma_{3}=\frac{3}{4}, \\
\rho_{1}=-\frac{17}{60}, & \rho_{2}=-\frac{5}{12}, &
\end{array}
$$


Evidently, at each time substep we should invert a banded matrix with the computation being carried on in the $y$-direction line by line, and the integral in $N(u)$ is evaluated at the beginning of each substep. A sponge is set to reduce the reflection at the boundaries in $x$ direction. Finally, we present the numerical boundary conditions as follows

$$
\begin{array}{r}
u_{x x}=0 \quad \text { at } \quad x=0, r_{x}, \\
\left.u_{y}\right|_{y=-r_{y}}=\left.u_{y}\right|_{y=+r_{y}},
\end{array}
$$

where $\left[0, r_{x}\right] \times\left[-r_{y}, r_{y}\right]$ is the computational domain.

\section{Numerical experiments and comparisons with exact solu- tions}

To illustrate the effectiveness of our numerical schemes for the KPI equation, the evolution of one lump soliton travelling in the $x$-direction is first investigated.

\subsection{Propagation of one lump soliton}

We computed solutions of the KPI equation subject to the following initial conditions

$$
u(x, y, 0)=16 \frac{-4\left(x-x_{0}\right)^{2}+16 k_{I}^{2}\left(y-y_{0}\right)^{2}+1 / k_{I}^{2}}{\left[4\left(x-x_{0}\right)^{2}+16 k_{I}^{2}\left(y-y_{0}\right)^{2}+1 / k_{I}^{2}\right]^{2}},
$$

with $k_{I}=\sqrt{6} / 4, x_{0}=15.0, y_{0}=0$. This means the lump soliton (the maximum of the lump is initially located at $\mathrm{x}=15.0$ ) will move to the positive $x$-direction with velocity $V_{x}=12 k_{I}^{2}=4.5$. Our computation was carried out in a rectangle $[0,50] \times[-20,20]$ with spatial step $[\triangle x, \triangle y]=[0.1,0.1]$ and time step $\triangle t=0.0002$. Fig. 1 shows the numerical solution from $t=0$ to $t=3$, respectively. Stable propagation of the soliton without any deformation is clearly seen. The conserved quantities of the KPI equation are also computed, and verified.

\subsection{Direct Interaction of two lump solitons}

In this section, the direct collision of two lump solitons with different velocities and phase were examined numerically and theorectically. We adopt the following initial conditions:

$$
\begin{gathered}
u(x, y, 0)=16 \frac{-4\left(x-x_{01}\right)^{2}+16 k_{1 I}^{2}\left(y-y_{01}\right)^{2}+1 / k_{1 I}^{2}}{\left[4\left(x-x_{01}\right)^{2}+16 k_{1 I}^{2}\left(y-y_{01}\right)^{2}+1 / k_{1 I}^{2}\right]^{2}}+ \\
16 \frac{-4\left(x-x_{02}\right)^{2}+16 k_{2 I}^{2}\left(y-y_{02}\right)^{2}+1 / k_{2 I}^{2}}{\left[4\left(x-x_{02}\right)^{2}+16 k_{2 I}^{2}\left(y-y_{02}\right)^{2}+1 / k_{2 I}^{2}\right]^{2}}
\end{gathered}
$$

where $y_{01}=y_{02}=0$, which implies two lump solitons moving along the same line. Feng \& Mitusi [6] and later Cao, Djidjeli and Price et al. [5] reported that two such lump solitons undergo an 'inelastic collision', i.e., they interact with each other and separate in the $y$ direction after the collision, forming two lumps of equal amplitude. However, as pointed out by Gorshkov et al. [10], these two separated solitons will undergo a striking process, 
i.e. they will come back together due to the nonlinear interaction, and then propagate in the $x$-direction, becoming again two lump solitons moving in the same $x$-direction. To illustrate this interesting phenomena and study the maximum separation in the $y$-direction, many calculations with different parameters have been carried out. Typical phenomena in the direct interaction of two lump solitons are illustrated in Fig. 2, 3 and 4, where Fig. 2 shows the initial condition with parameters $x_{01}=15, x_{01}=31, k_{1 I}=\sqrt{6} / 4, k_{2 I}=\sqrt{6} / 8$, and Fig. 3 shows the profiles of two solitons from $t=2.5$ to $t=7.5$ with increment of 1.0 . Figure 4 shows contours of two solitons from time $t=1$ to $t=12$ with interval $\triangle t=1$. The present numerical results confirm the results of Gorshkov et al [10], and from them it is seen that when the tall one approaches the small one, the amplitude and velocity of the tall one increase while those of the small one decrease, and at the same time, the small one separates in $y$-direction, As time increases, they evolve into two lumps of equal amplitude moving in opposite $y$-directions; after reaching their maximum separation in the $y$ - direction they come back to the $x$-axis, and then evolve to two lump solitons moving in the $x$-direction again. Now the tall one is in front of the small one, and as time goes on, these two solitons will recover to their initial states (which is easily proven from the exact solution by symbolic computation).

The exact solution in this case is shown in Fig. 5, and it is obviously seen that our numerical results compare well with the exact one. Fig. 6 shows the trajectory of two lumps before and after separation. Among them Fig. 6a shows the path of each lump soliton where the solid line means the path of each soliton when ignoring the existence of the other one (then the speed is constant) and Fig. 6b is the corresponding trajectory in the $X-Y$ plane. From these figures it is clearly shown that the tall (small) lump moves faster (slower) than its own velocity (the velocity when totally ignoring the existence of the other one) before collision, whilst the tall (small) one moves slower (faster) after collision. This means two solitons attract each other before collision and repel after collision. From Fig. 7 , which shows the contours of two solitons from the exact solution (6) with parameters $k_{1 R}=k_{2 R}=\gamma_{1 I}=\gamma_{2 I}=0, \gamma_{01}=15, \gamma_{02}=31, k_{1 I}=\sqrt{6} / 4, k_{2 I}=\sqrt{6} / 12$ at $t=1$ to $t=10$ with interval $\triangle t=1$, we can see clearly that two solitons don't repel in the $y$-direction. Our numerical results determine that whether there is separation or not, and the maximum separation distance in the $y$-direction, depends on the initial relative velocity difference, but scarcely depends on the initial phase (i.e. initial position of two lumps).

To quantitatively study this dependence, we have to turn to the exact solution. Direct interaction means we should set: $k_{1 R}=k_{2 R}=\gamma_{1 I}=\gamma_{2 I}=0$ in Eq.6. First, we investigate the phase change before and after the nonlinear interaction. With the help of symbolic computation, it is easily proved that their phase shifts turn out to be zero after collision, with also their amplitude, velocity and size are restored. Secondly we investigate quantitatively the largest vertical distance between two solitons. Due to independence of the distance on the initial phase, it is, for brevity, further assumed that $\gamma_{1 R}=\gamma_{2 R}=0$. With the help of symbolic computation again, we finally formulate the maximum separation in the $y$ - direction in terms of the velocities $\left(V_{1}=V_{1 x}, V_{2}=V_{2 x}\right)$

$$
\begin{aligned}
y_{m}= & 6\left(\frac { 1 } { V _ { 1 } ^ { 3 } ( V _ { 1 } - V _ { 2 } ) ^ { 4 } V _ { 2 } ^ { 3 } } \left(-V_{1}^{6} V_{2}^{2}-12 V_{1}^{5} V_{2}^{3}+26 V_{1}^{4} V_{2}^{4}-12 V_{1}^{3} V_{2}^{5}-V_{1}^{2} V_{2}^{6}+\right.\right. \\
& \left(-V_{1}^{3}\left(V_{1}-V_{2}\right)^{4} V_{2}^{3}\left(V_{1}^{6}+10 V_{1}^{5} V_{2}-113 V_{1}^{4} V_{2}^{2}-\right.\right. \\
& \left.\left.\left.\left.820 V_{1}^{3} V_{2}^{3}-113 V_{1}^{2} V_{2}^{4}+10 V_{1} V_{2}^{5}+V_{2}^{6}\right)\right)^{1 / 2}\right)\right)^{1 / 2}
\end{aligned}
$$


let $V_{1}=(k+1) V_{2}$, i.e. $\left(V_{1}-V_{2}\right) / V_{2}=k$, then we can get

$$
\begin{aligned}
y_{m}= & \frac{6}{k V_{2}}\left(\frac { - 1 } { ( 1 + k ) ^ { 3 } } \left(16+48 k+49 k^{2}+18 k^{3}+k^{4}-\right.\right. \\
& \left.\left.\left((1+k)^{3}\left(1024+3072 r+3136 k^{2}+1152 k^{3}+48 k^{4}-16 k^{5}-k^{6}\right)\right)^{1 / 2}\right)\right)^{1 / 2}
\end{aligned}
$$

when $k \rightarrow 0$ in Eq. (14), it reads

$$
y_{m}=\frac{24}{k V_{2}}
$$

Fig. 8 shows the dependence of $y_{m} V_{2}$ on the relative ratio $k$ and the limit behavior when $k \rightarrow 0$, from which we can get the critical relative velocity difference $k_{c}=7.326$. It has been confirmed by the numerical results shown in Fig. 7 where $k=8$, i.e., no separation occurs.

\subsection{Oblique Interaction of two lump solitons}

There are two types of oblique interaction. When $V_{1 y}$ and $V_{2 y}$ have the opposite sign we call them, oblique collision I, and when they possess the same sign, oblique collision II. To illustrate these interaction processes, we give three numerical examples, where without loss of generality, $k_{l R}=k_{1 I}, i=1,2$ are imposed in each example.

1. Case 1

The initial condition is as follows:

$$
\begin{aligned}
u(x, y, 0)= & 16 \frac{-4\left(x-x_{01}-2 k_{1 I}\left(y-y_{01}\right)\right)^{2}+16 k_{1 I}^{2}\left(y-y_{01}\right)^{2}+1 / k_{1 I}^{2}}{\left[\left(x-x_{01}-2 k_{1 I}\left(y-y_{01}\right)\right)^{2}+16 k_{1 I}^{2}\left(y-y_{01}\right)^{2}+1 / k_{1 I}^{2}\right]^{2}}+ \\
& 16 \frac{-4\left(x-x_{02}-2 k_{2 I}\left(y-y_{02}\right)\right)^{2}+16 k_{2 I}^{2}\left(y-y_{02}\right)^{2}+1 / k_{2 I}^{2}}{\left[4\left(x-x_{02}-2 k_{2 I}\left(y-y_{02}\right)\right)^{2}+16 k_{2 I}^{2}\left(y-y_{02}\right)^{2}+1 / k_{2 I}^{2}\right]^{2}}
\end{aligned}
$$

where $k_{1 I}=0.5, k_{2 I}=-0.5$, and $x_{01}=15, y_{01}=-15, x_{02}=15, y_{02}=15$. The initial profile is shown in Fig 9. from which we see that two solitons with the same amplitude are initially independently, located symmetrically about the $x$-axis. In Fig. 10, we plot contours at $t=1$ to $t=10$ from the numerical results. These show that two solitons move without noticing the existence of the other one. The exact solution confirms that two solitons moving from opposite $y$-directions to the horizontal do move along their original paths. This is true even when the two solitons have different amplitudes(velocities). Of course, when they interact they are far from just a linear superposition of two independent solitons.

2. Case 2

In this case, the initial condition is the same as Eq. (16), but with parameters $k_{1 I}=$ $0.5, k_{2 I}=0.3$, and $x_{01}=15, y_{01}=-30, x_{02}=34.2, y_{02}=-18$. which means the two solitons initially are located in the same side of the $x$-axis, and they will travel to the same position $(45,0)$. The initial profile is shown in Fig.11. The numerical results are shown in Fig. 12, while the exact solution with the same parameters is shown in Fig. 13. The agreement between numerical and exact solutions is quite satisfactory. We can see that when the two solitons approach together from the same side, the tall one gradually reduces its amplitude and velocity, while the small one increases its amplitude and velocity, and finally the two solitons become of the same size at the minimum distance apart (this distance cannot be zero unless the relative amplitude difference of two solitons is large enough). After that, the two solitons interchange their positions, this means two solitons experience 
an abrupt phase change. Due to the nonlinear interaction, the front soliton becomes taller until it recovers its initial amplitude, while the rear soliton decreases and gradually recover its original state. Fig. 14 shows the trajectory of two lumps in the oblique collision. Again the solid line shows the trajectory of each soliton when ignoring the existence of the other one. It is clearly seen that the two solitons attract each other before collision and repel after collision, and both solitons experience an abrupt phase shift. Another interesting phenomena in this case is when the relative amplitude difference between the two solitons is large enough, two solitons can merge and then separate and move in their own original ways. Fig. 15 shows the exact solution with parameters $k_{2 I}=0.1$ and the other parameters at the same. It seems that the interaction between two solitons strongly depends on their relative amplitude difference, and strongly nonlinear interactions can only occur when this difference becomes larger than a critical value.

\section{Conclusions}

The interaction of two lump solitons described by the KPI equation has been analyzed using exact and numerical methods. The numerical method adopted here is the combination of 3order Runge-Kutta method and the Crank-Nicolson scheme. The comparison of numerical results with the exact solutions is satisfactory, and shows the numerical method is effective. Some rich phenomena of lump soliton interactions are presented. The most interesting property revealed in this paper is that the details of the interaction strongly depend on the relative velocity (or amplitude) difference and the way in which two solitons are approaching; i.e. they may separate in the $y$ - direction in a direct interaction with the moderate relative velocity difference, or no separation occurs for a large velocity difference (larger than a critical value, which is approximately 7.326); they may exchange their positions in oblique collision(case 2 with moderate relative amplitude difference), or almost keep their original ways seeming to show total ignorance of the existence of the other lump(case 1 and case 2 with big relative amplitude difference).

\section{Acknowledgement}

Constructive discussions with Dr. Dmitry Pelinovskii at the Department of Mathematics of MacMaster University in Canada are greatly appreciated. Z.Lu would also like to thank the hospitality of Department of Mathematics and Statistics at Wright State University where part of this work was carried out.

\section{References}

[1] M.J. Ablowitz and P.A. Clarkson, Solitons, Nonlinear Evolution Equations and Inverse Scattering. Cambridge University Press, Cambridge (1991).

[2] M.J. Ablowit and J. Villarroel, Solutions to the time dependent Schrödinger and the Kadomtsev- Petviashvili equations. Phy. Rev. Letters. 78(4),570- 574 (1997) 
[3] K. M.Berger and P. A. Milewski, The Generation and evolution of lump solitary waves in surface- tension-dominated flows. SIAM J. Appl. Math.. 61(3), 731-750 (2000)

[4] A. G. Bratsos and E. H. Twizell, An explicit finite difference scheme for the solution of Kadomtsev-Petviashvili equation. Int. J. Comput. Math..68,175-187 (1998)

[5] Q.Cao, K.Djidjeli, W.G.Price and E.H.Twizell, Computational methods for some nonlinear wave equations Journal of Engineering Mathematics, 35, 323-338 (1999)

[6] B.F. Feng and T. Mitsui, A finite difference method for the Korteweg-de Vries and the Kadomtsev-Petviashvili equations. J. Comp.\&Appl. quadMath.. 90, 95-116 (1998)

[7] A.S. Fokas, D.E. Pelinovsky and C. Sulaem, Interaction of lumps with a line soliton for the DSII equation. Physica D. 152-153, 189-198 (2001)

[8] N.C. Fornberg and G.B. Whitham, A numerical and theorectical study of certain nonlinear wave phenomena. Phil. Trans. Roy. Soc. London. 289, 373-404 (1978)

[9] D.J. Frantzeskakis, K. Hizanidis, B.A. Malomed, A. Polymilis, Stable anti-dark light bullets supported by the third-order dispersion. Physics Letters A. 248, 203-207 (1998)

[10] K.A. Gorshkov, D. E. Pelinovsky and Yu. A Stepanyants, Normal and anormal scattering, formation and decay of bound states of two-dimensional solitons described by the Kadomtsev- Petviashvili equation. JETP. 77(2), 237-245 (1993)

[11] E.Infeld, A.Senatorski, and A.A.Skorupski, Numerical simulations of KadomtsevPetviashvili soliton interactions Physical Review E, 51(4), 3183-3191 (1995)

[12] B. B. Kadomtsev and V. I. Petviashvili, On the stability of solitary waves in weakly dispersing media. Soviet Phys. Dockl. 15, 539-541 (1970)

[13] C. Katsis and T.R. Akylas, On the excitation of long nonlinear water waves by a moving pressure distribution. Part 2. Three-dimensional effects. J. Fluid Mech.. 177, 49-65 (1987)

[14] S. V. Manakov, V.E. Zakhorov and L.A. Bordag et al., Two-dimensional solitons of the Kadomtsev-Petviashvili equation and their interaction Phys. Lett., 63, 205-206 (1977)

[15] A.A.Minzoni, N.F.Smyth, Evolution of lump solutions for the KP equation Wave Motion, 24, 291-305 (1996)

[16] D.E. Pelinovsky and Yu. A. Stepanyants, New multisoliton solutions of the KadomtsevPetviashvili equation. JETP Letter. 57(1), 24-28 (1993)

[17] C.Polymilis, D.J.Frantzeskakis, and A.N. Yannacopoulos, Optical stripes and bullets for a modified nonlinear Schrödinger equation. J. Opt. Soc. Am. B, 18, 75-80 (2001)

[18] A.-M. Wazwaz, A computational approach to soliton solutions of the Kadomtsev- Petviashvili equation. Appl.Math.Comput., 123,205-217 (2001) 


\section{List of Figures}

1 The propagation of one soliton moving on the line $y=0 . t=1,2,3 a: t=0.0, b$ : $\mathrm{t}=1.0, \mathrm{c}: \mathrm{t}=2.0, \mathrm{~d}: \mathrm{t}=3.0 ; \ldots \ldots \ldots \ldots$

2 The initial profile of two solitons on the line $\mathrm{y}=0$ with parameters $k_{1 I}=$ $\sqrt{6} / 4, k_{1 I}=\sqrt{6} / 8 \ldots \ldots \ldots \ldots \ldots \ldots \ldots \ldots \ldots \ldots \ldots \ldots \ldots \ldots$

The profile of two solitons in a direct interaction at $\mathrm{t}=2.5$ to 7.5 with increment of $1.0 \mathrm{a}: \mathrm{t}=2.5 \mathrm{~b}: \mathrm{t}=3.5, \mathrm{c}: \mathrm{t}=4.5, \mathrm{~d}: \mathrm{t}=5.5$, e: $\mathrm{t}=6.5, \mathrm{f}: \mathrm{t}=7.5 \ldots$

4 The contours of two solitons in a direct interaction(numerical solution) from $\mathrm{t}=1.0$ to 12.0 left (top to bottom): $\mathrm{t}=1.0,2.0, \ldots 6.0$; right (top to bottom): $\mathrm{t}=7.0,8.0, \ldots 12.0 \ldots \ldots \ldots \ldots \ldots \ldots$

5 The contours of two solitons in a direct interaction (exact results) $t=1.0$ to 6.0 from top left to down left; $\mathrm{t}=7$ to 12.0 from top right to down right . .

6 The trajectories of two lumps in direct collision. top: the path of the tall lump where the solid line represents the path when totally ignoring the existence of the small lump, while the dots show the position at $\mathrm{t}=0,0.5, \ldots, 3.0$; $6.0,6.5, \ldots, 9.0$; middle: the path of the small lump where the solid line represents the path when totally ignoring the existence of the tall lump, while the dots show the position at $\mathrm{t}=0,0.5, \ldots, 3.0 ; 6.0,6.5, \ldots, 9.0$; bottom: the trajectories in $X$-Y plane: diamond the tall lump, star the small lump, square two separated lumps in $y$-direction . . . . . . . . . . . . . . .

7 The contours of two solitons (exact solutions) subject to initial condition as: $k_{2 I}=\sqrt{6} / 12$ at $\mathrm{t}=1,2, \ldots, 5$ (left from top to bottom); $6,7, \ldots, 10$ (right from top to bottom) . . . . . . . . . . . . . . . .

8 The dependance of the maximum separation of two solitons in the case of a direct collison on the relative velocity difference, where the solid line is the exact formula, and the dash line is the limiting behaviour when $\mathrm{k}$ goes to zero. 16

9 The initial condition of two solitons in an oblique collision (case 1) . . . .

10 The contours of two solitons in an oblique interaction (case 1) (Numerical solution) at $\mathrm{t}=1,2, \ldots, 5$ (left from top to bottom); $6,7, \ldots, 10$ (right from top to bottom) . . . . . . . . . . . . . . . .

11 The initial condition of two solitons in an oblique collision (case 2) . . .

12 The contours of two solitons in an oblique interaction (case 2 ) at $t=0,1, \ldots 9$ (Numerical solution) . . . . . . . . . . . . . . . .

13 The contours of two solitons in an oblique interaction (case 2)(Exact solution) at $\mathrm{t}=1,3,5,7,9$ (from top to bottom) . . . . . . . . . . . . . .

14 The trajectories of two lumps in an oblique interaction. top the path of the tall lump where the solid line means the path when ignoring the existence of the small lump, while the dots represent the location at $\mathrm{t}=0.0,0.5, \ldots, 4.5$; $5.5,6.0, \ldots, 9.5$; bottom the path of the small lump where the solid line means the path when ignoring the existence of the tall lump while the dots represent the location at $\mathrm{t}=0.0,0.5, \ldots, 4.5 ; 5.5,6.0, \ldots, 9.5 \ldots \ldots \ldots$

15 The contours of two solitons(exact solution) with parameters $k_{1 I}=k_{1 R}=$ $0.5, k_{2 I}=k_{2 R}=0.1$ at $\mathrm{t}=1,3,5,7,9$ (from top to bottom) $\ldots \ldots \ldots$ 


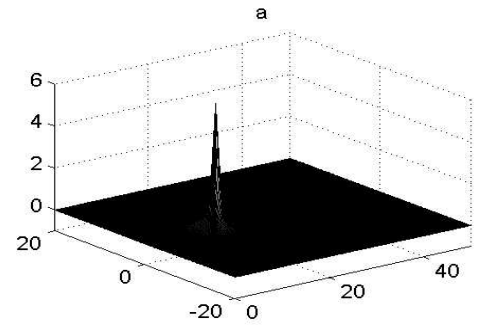

b

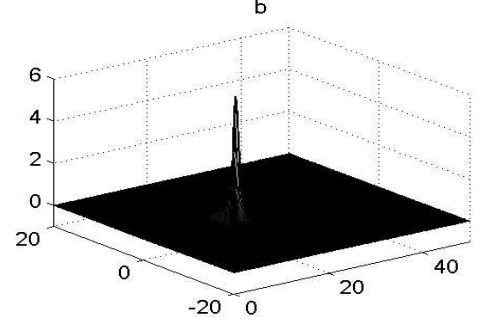

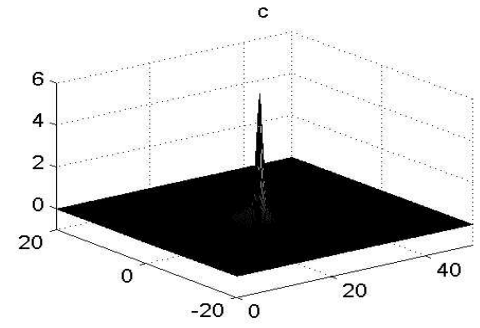

d

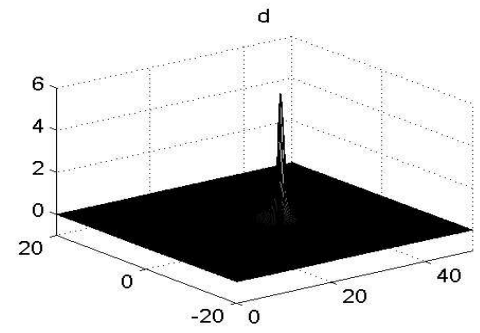

Figure 1: The propagation of one soliton moving on the line $y=0 . t=1,2,3 a: t=0.0, b$ : $\mathrm{t}=1.0, \mathrm{c}: \mathrm{t}=2.0, \mathrm{~d}: \mathrm{t}=3.0$

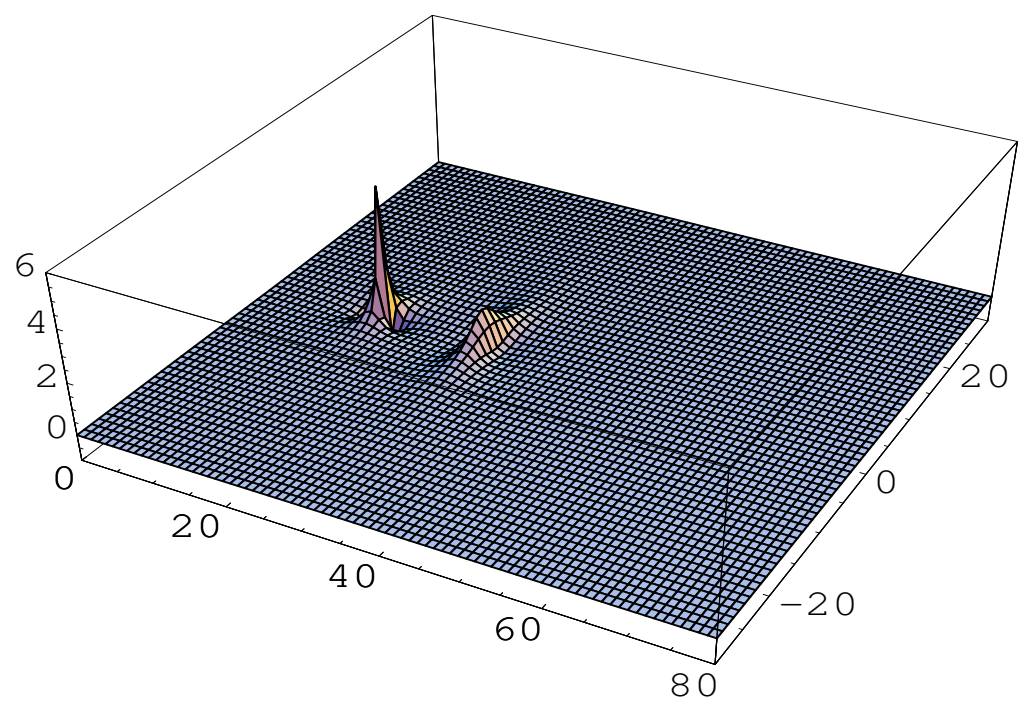

Figure 2: The initial profile of two solitons on the line $\mathrm{y}=0$ with parameters $k_{1 I}=$ $\sqrt{6} / 4, k_{1 I}=\sqrt{6} / 8$ 

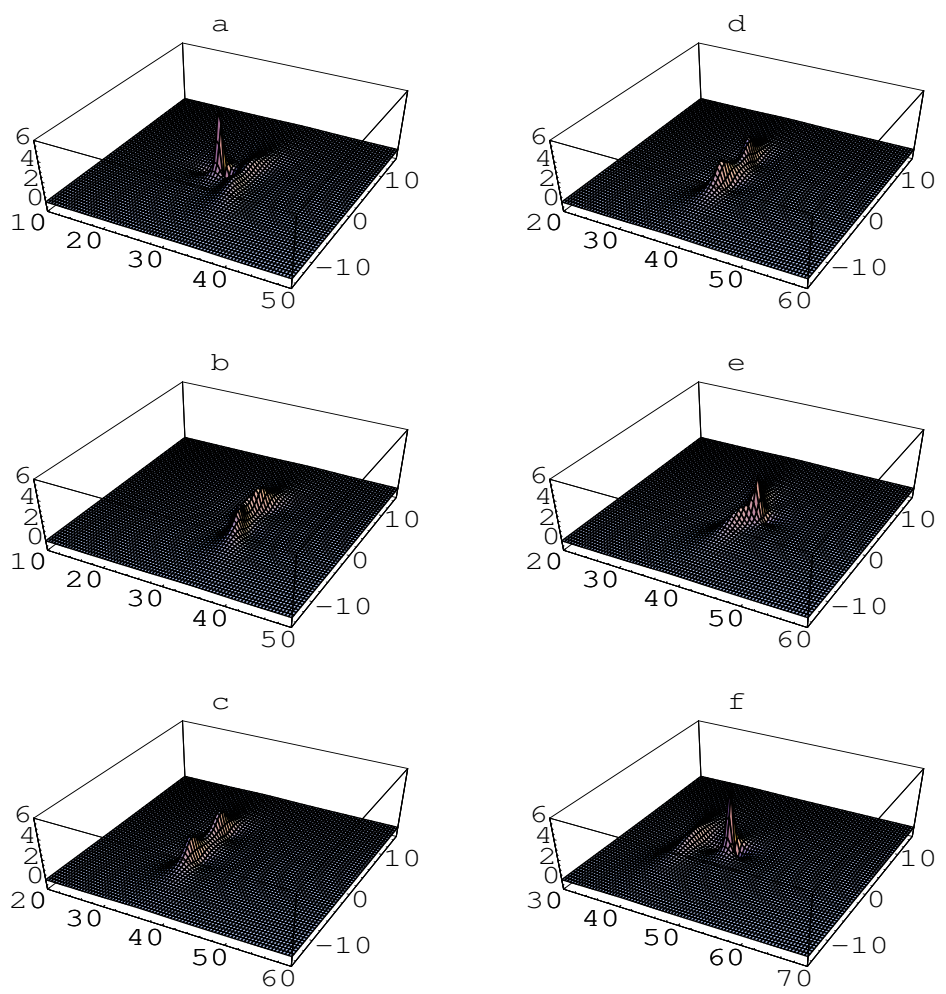

Figure 3: The profile of two solitons in a direct interaction at $\mathrm{t}=2.5$ to 7.5 with increment of $1.0 \mathrm{a}: \mathrm{t}=2.5 \mathrm{~b}: \mathrm{t}=3.5, \mathrm{c}: \mathrm{t}=4.5, \mathrm{~d}: \mathrm{t}=5.5$, e: $\mathrm{t}=6.5, \mathrm{f}: \mathrm{t}=7.5$ 

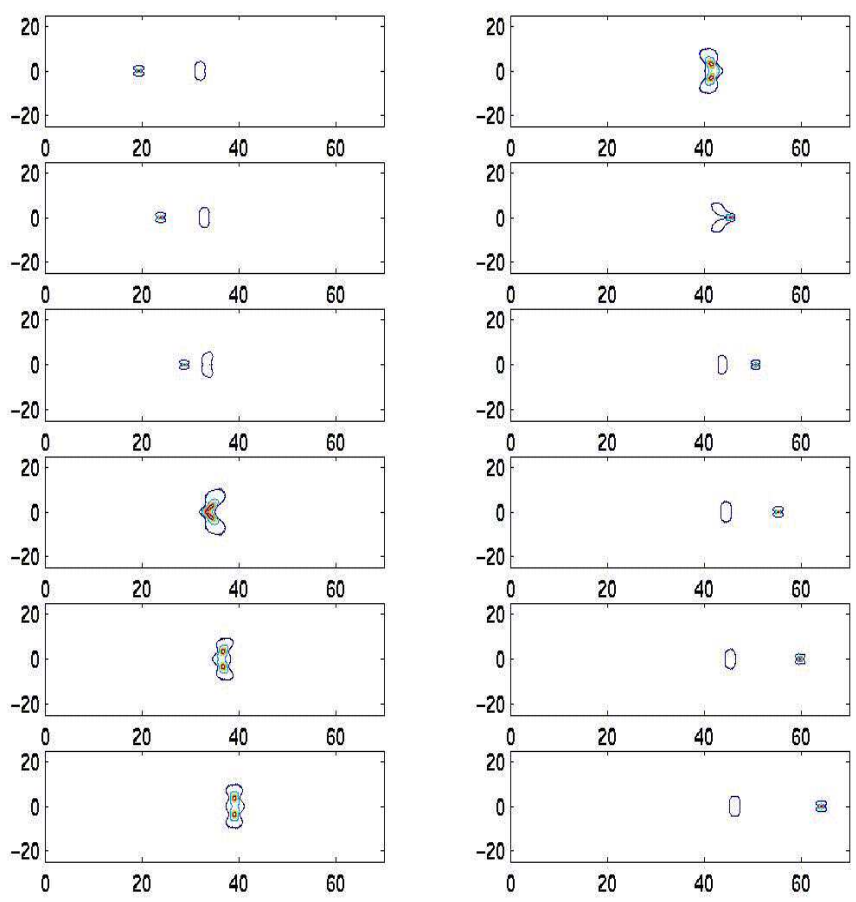

Figure 4: The contours of two solitons in a direct interaction(numerical solution) from $\mathrm{t}=1.0$ to 12.0 left (top to bottom): $\mathrm{t}=1.0,2.0, \ldots 6.0$; right (top to bottom): $\mathrm{t}=7.0,8.0, \ldots 12.0$ 

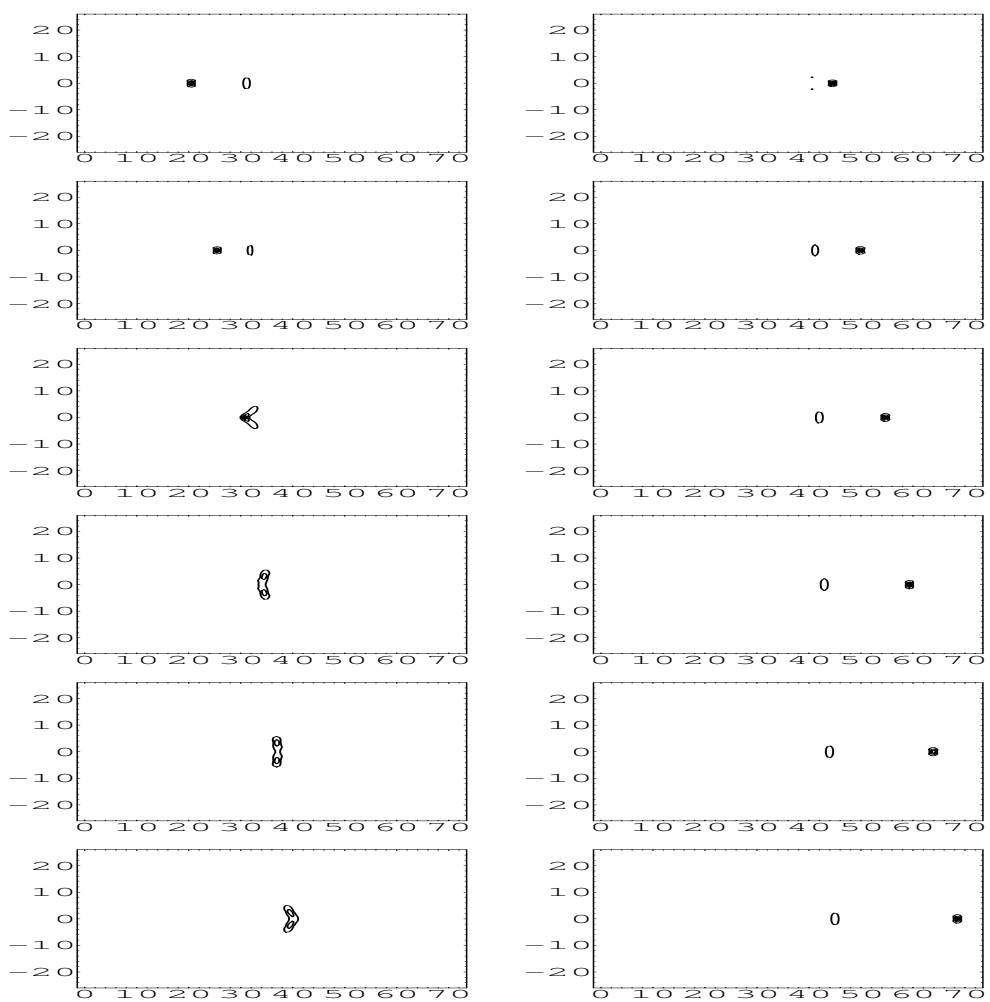

Figure 5: The contours of two solitons in a direct interaction (exact results) $t=1.0$ to 6.0 from top left to down left; $\mathrm{t}=7$ to 12.0 from top right to down right 

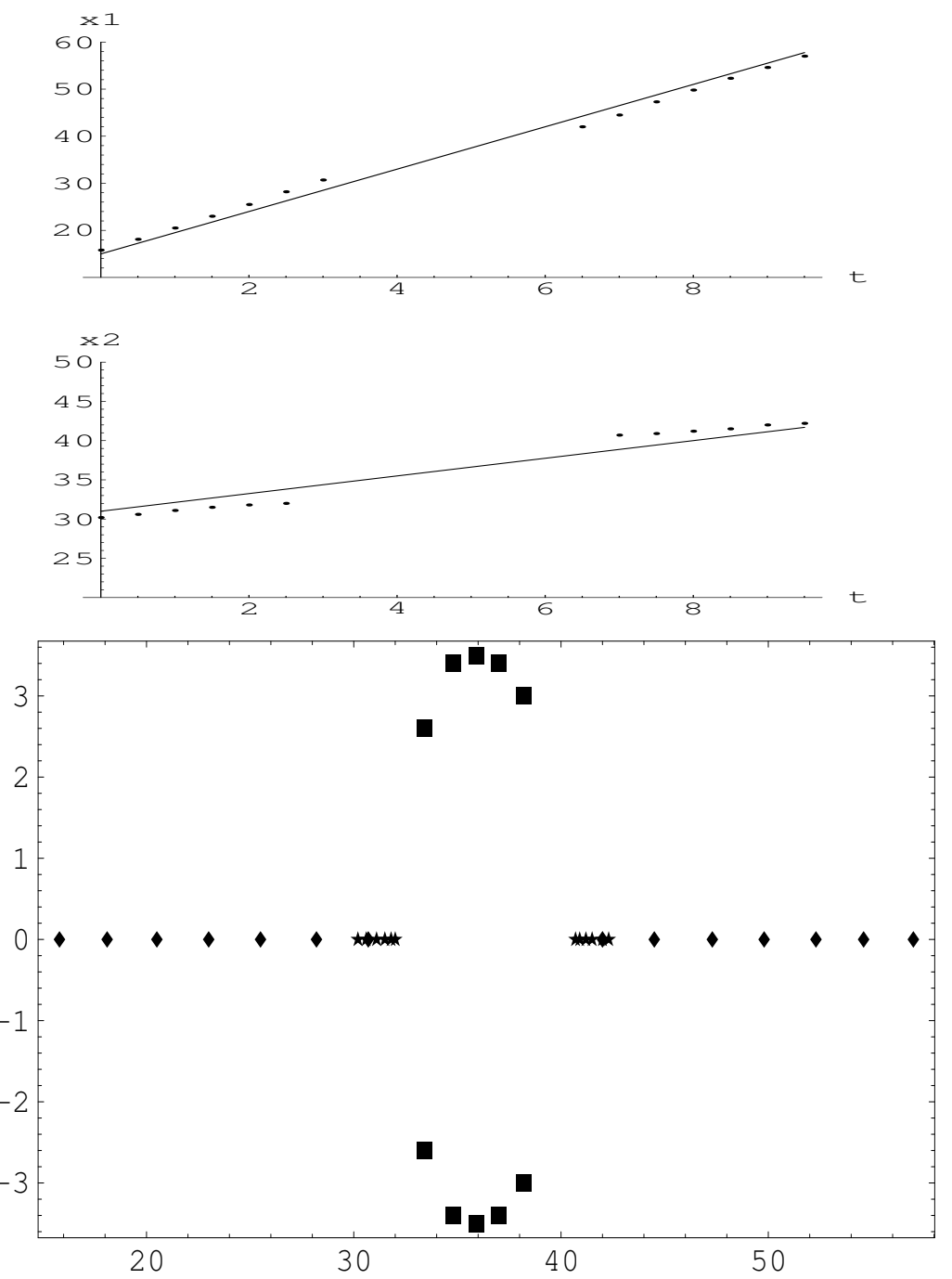

Figure 6: The trajectories of two lumps in direct collision. top: the path of the tall lump where the solid line represents the path when totally ignoring the existence of the small lump, while the dots show the position at $\mathrm{t}=0,0.5, \ldots, 3.0 ; 6.0,6.5, \ldots, 9.0 ;$ middle: the path of the small lump where the solid line represents the path when totally ignoring the existence of the tall lump, while the dots show the position at $\mathrm{t}=0,0.5, \ldots, 3.0 ; 6.0,6.5, \ldots, 9.0 ;$ bottom: the trajectories in $X$-Y plane: diamond the tall lump, star the small lump, square two separated lumps in $y$-direction 

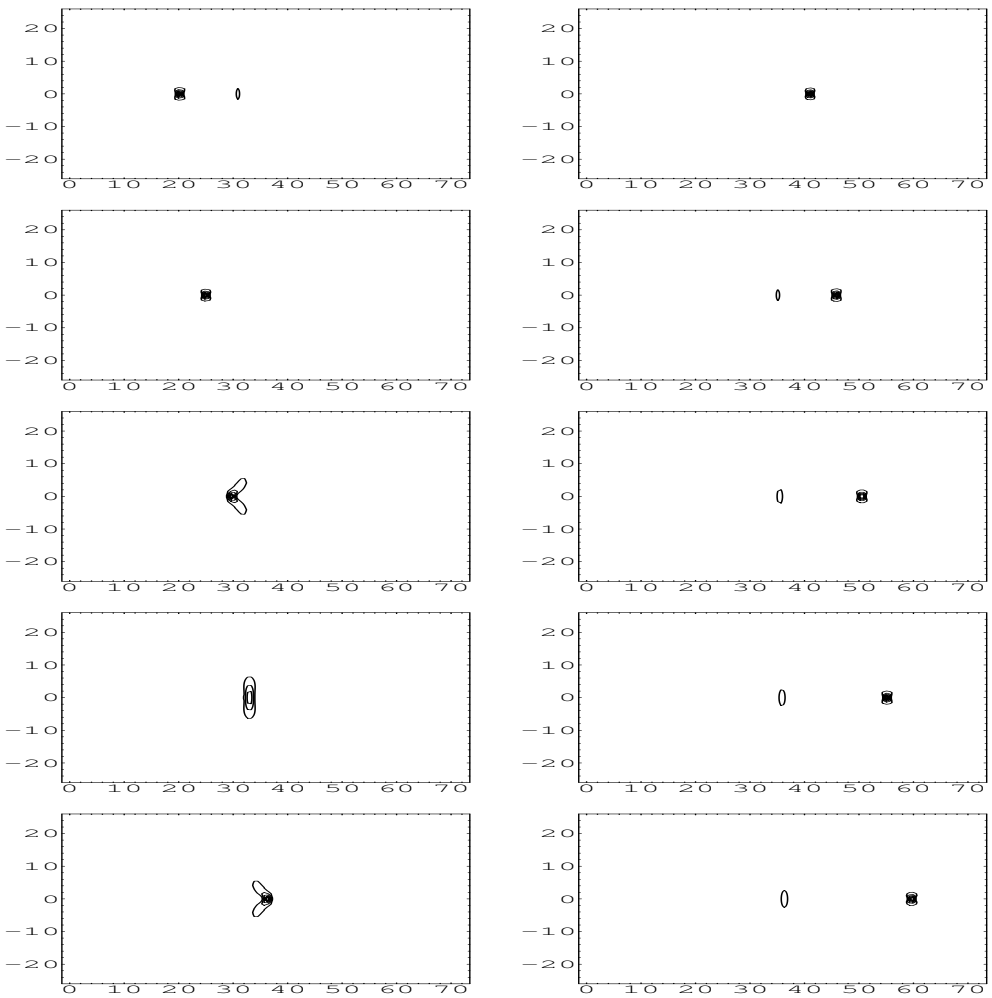

Figure 7: The contours of two solitons (exact solutions) subject to initial condition as: $k_{2 I}=\sqrt{6} / 12$ at $\mathrm{t}=1,2, \ldots, 5$ (left from top to bottom); $6,7, \ldots, 10$ (right from top to bottom)

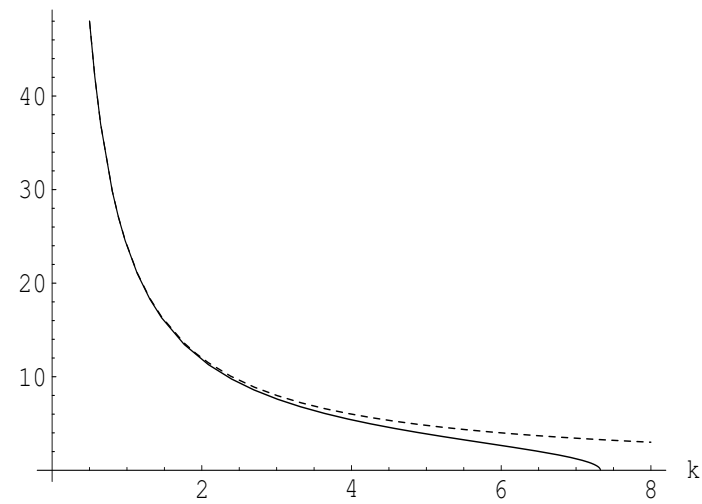

Figure 8: The dependance of the maximum separation of two solitons in the case of a direct collison on the relative velocity difference, where the solid line is the exact formula, and the dash line is the limiting behaviour when $\mathrm{k}$ goes to zero. 


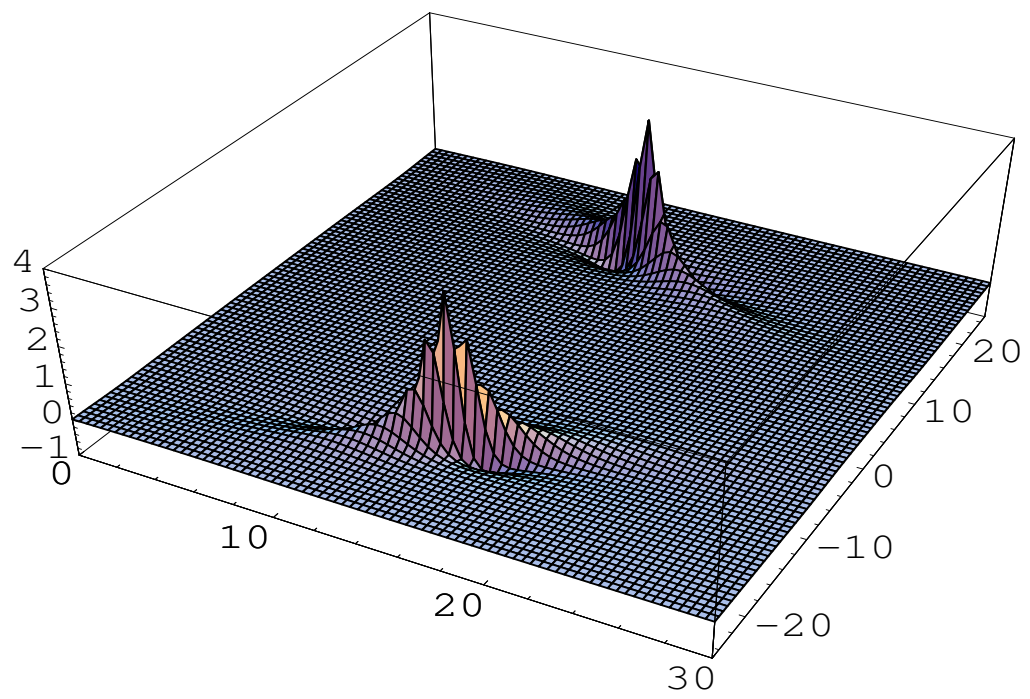

Figure 9: The initial condition of two solitons in an oblique collision (case 1)
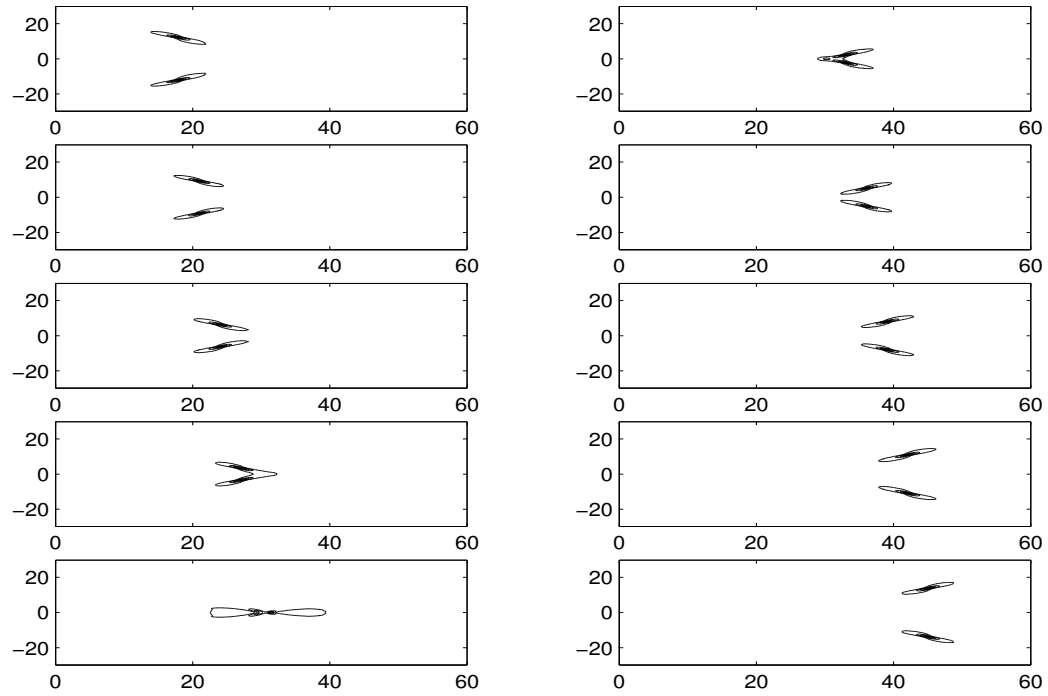

Figure 10: The contours of two solitons in an oblique interaction (case 1) (Numerical solution) at $\mathrm{t}=1,2, \ldots, 5$ (left from top to bottom); $6,7, \ldots, 10$ (right from top to bottom) 


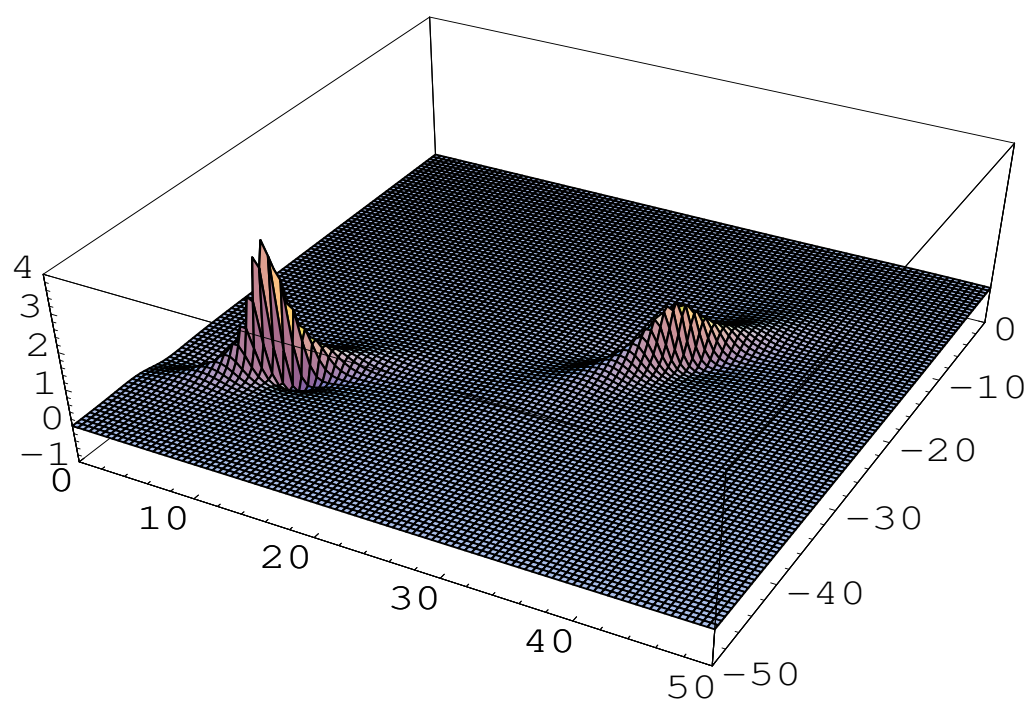

Figure 11: The initial condition of two solitons in an oblique collision (case 2)
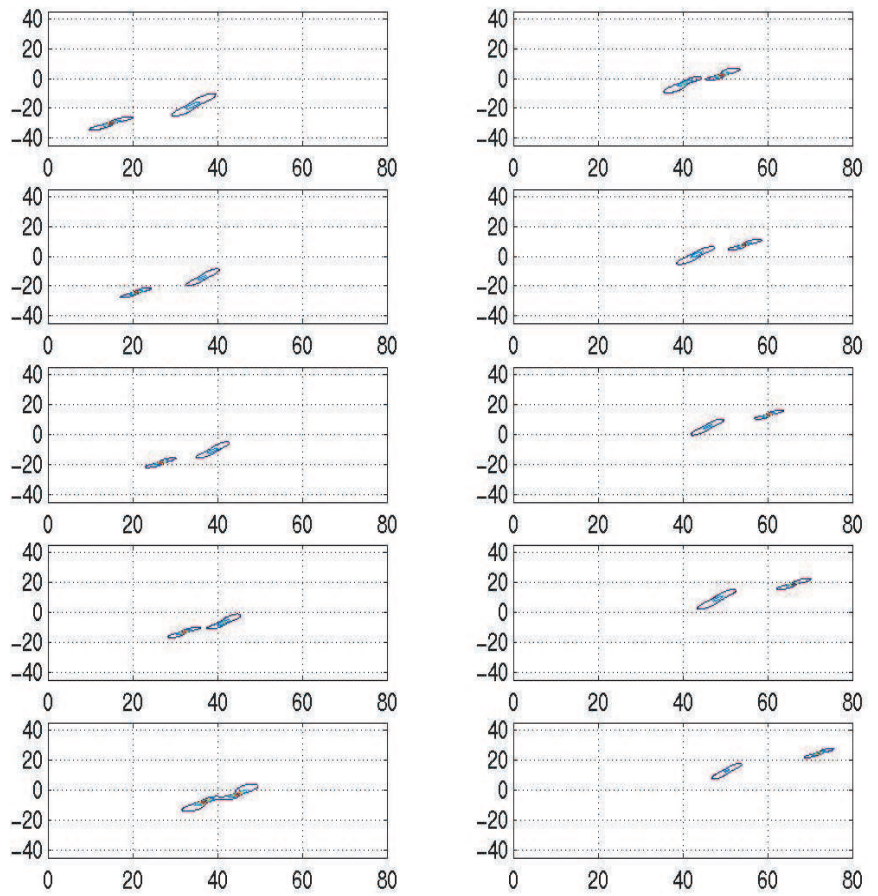

Figure 12: The contours of two solitons in an oblique interaction (case 2 ) at $t=0,1, \ldots 9$ (Numerical solution) 


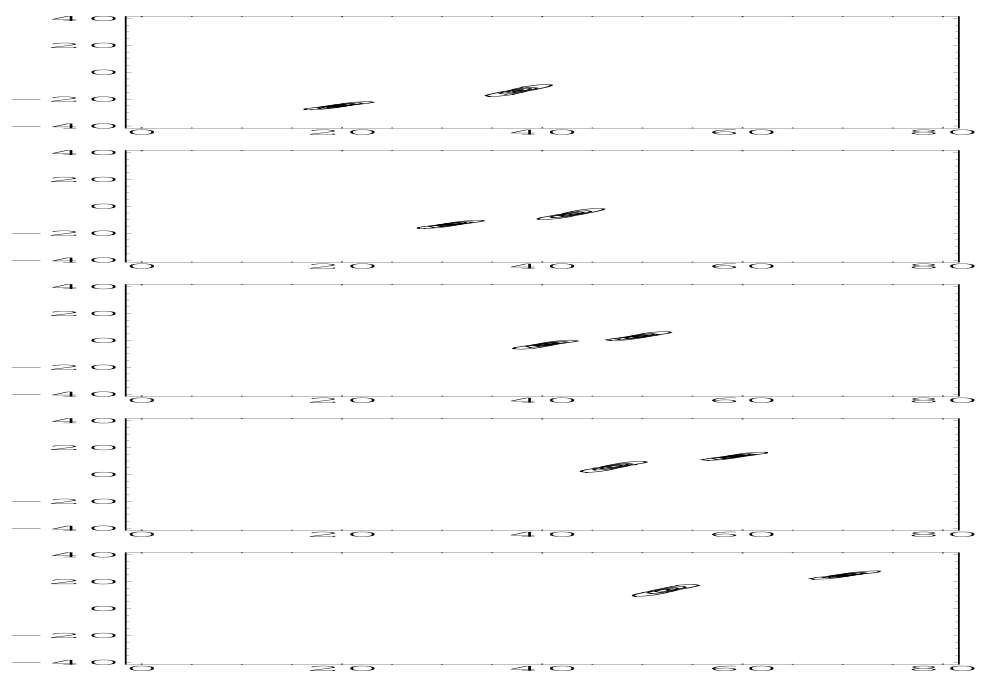

Figure 13: The contours of two solitons in an oblique interaction (case 2)(Exact solution) at $\mathrm{t}=1,3,5,7,9$ (from top to bottom)
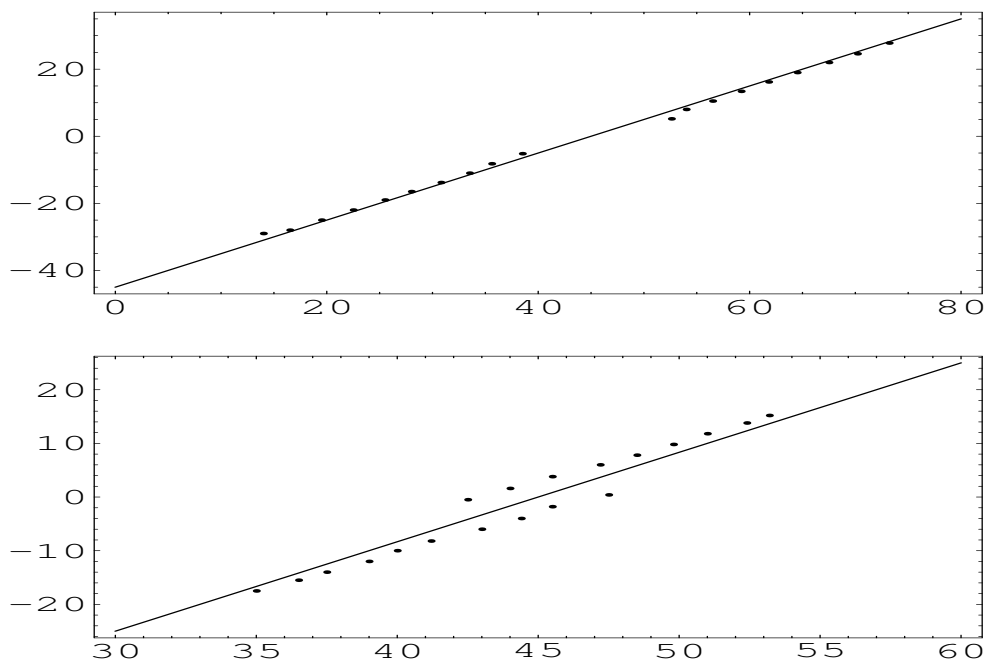

Figure 14: The trajectories of two lumps in an oblique interaction. top the path of the tall lump where the solid line means the path when ignoring the existence of the small lump, while the dots represent the location at $\mathrm{t}=0.0,0.5, \ldots, 4.5 ; 5.5,6.0, \ldots, 9.5$; bottom the path of the small lump where the solid line means the path when ignoring the existence of the tall lump while the dots represent the location at $\mathrm{t}=0.0,0.5, \ldots, 4.5 ; 5.5,6.0, \ldots, 9.5$ 

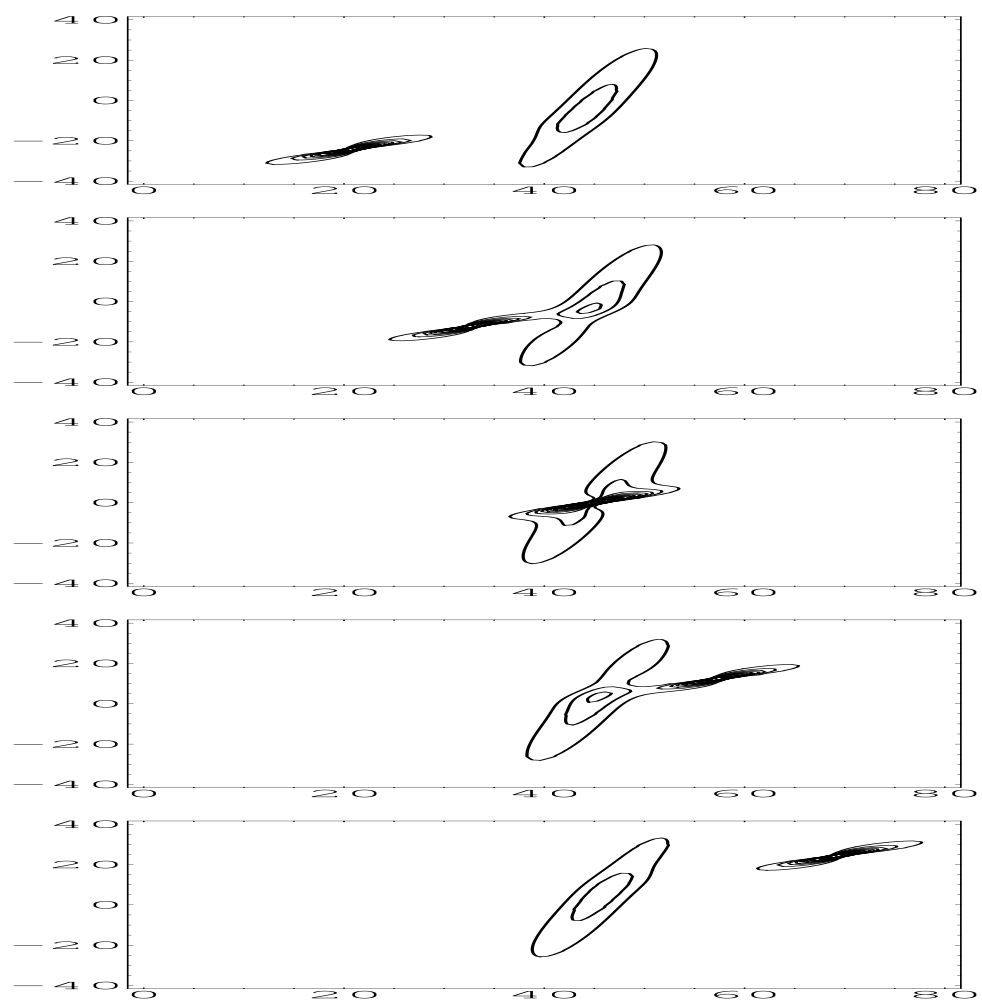

Figure 15: The contours of two solitons(exact solution) with parameters $k_{1 I}=k_{1 R}=$ $0.5, k_{2 I}=k_{2 R}=0.1$ at $\mathrm{t}=1,3,5,7,9$ (from top to bottom) 